\title{
Maternal Intravenous Fluids and Infant Weight
}

Share this: $\mathbf{f} \boldsymbol{E}$

\begin{abstract}
Healthcare providers typically use an infant's weight loss in the first days of life as a measurement of effective feeding. Additional feeding volumes are often recommended when the infant reaches weight loss of seven to ten percent of their birth weight. This study examined the relationship of the amount of maternal intravenous fluids (IV) given during labor, and infant maximum weight loss during hospital admission. The method was a retrospective cross-sectional review of medical records for 186 healthy mothers and their infants who delivered at a Baby Friendly ${ }^{\mathrm{TM}}$ certified hospital in southwest Ohio. Maternal average IV mL per hour positively correlated with infant maximum weight loss.
\end{abstract}

Keywords: infant weight loss, maternal IV fluids, labor Clinical Lactation, 2012, Vol. 3-2, 59-63

Healthcare providers typically use an infant's weight loss in the first few days of life as a measurement of effective feeding. Supplementation of breastfeeding or increased formula feeding is often recommended when the infant reaches weight loss of seven to ten percent of their birth weight (Academy of Breastfeeding Medicine, 2009; American Academy of Pediatrics, 2005; International Lactation Consultant Association, 2005; Mulder et al., 2010). The range of weight loss can be influenced by a variety of factors. However, minimal research has explored the potential influence of maternal intravenous (IV) volume received during labor.

\section{Influences on Infant Weight Loss after Birth}

Increased infant weight loss during the first few days of life in all infants is associated with female gender, epidural, primiparous mother, cesarean section, and feeding method (Martens \& Romphf, 2007). Increased infant weight loss in breastfed infants is associated with cesarean section (Saki et al., 2010), delayed onset of lactogenesis (Dewey et al., 2003), suboptimal breastfeeding, exclusive breastfeeding or breastfeeding with supplements (Chantry et al., 2011; Martens \& Romphf, 2007), labor > 14 hours (Dewey et al., 2003), multiparous women who receive labor pain medication (Dewey et al., 2003), and maternal breast variation (Vazirinejad et al., 2009).

Early questions about the effect of the volume of maternal IV fluid and infant weight loss were suggested in articles by Merry and Montgomery (2000) and Dewey

\footnotetext{
1. rlhirth@health-partners.org

2. Tina.Weitkamp@UC.edu

3. Alok_Bhul@yahoo.com
}

et al. (2003). The first study was a master's thesis by Sheehan (2009), which reported that a total maternal intravenous volume of $>1225 \mathrm{~mL}$ (regardless of duration of fluid intake), cesarean section, and epidural were significant for infant weight loss $\geq 7 \%$ of birth weight at 72 hours of age. Lamp and Macke (2010) studied intrapartum maternal fluid balance (IV fluid administered + oral intake). Infants were weighed at $48 \pm$ 2 hours, and $4.3 \%$ were found to have lost $\geq 10 \%$. They found that intrapartum maternal fluid balance was not significant for weight loss. However, type of feed ( $p=$ 0.000 ) and number of wet diapers were significant for weight loss $(p=0.003)$. Chantry et al. (2011) found that excessive weight loss was independently related to intrapartum fluid balance. The relative risk for excessive weight loss more than tripled with a maternal positive fluid balance exceeding $200 \mathrm{~mL} /$ hour when compared to $100 \mathrm{~mL} /$ hour.

Noel-Weiss et al. (2011) found that the timing and amounts of fluid (including oral intake and IV fluids) correlated with the output and weight loss of breastfed newborns. The authors observed a 24-hour post-birth period of newborn diuresis and fluid-balance correction. Therefore, they recommended that a newborn weight measurement at 24 hours replace birth weight as baseline for weight change assessment.

\section{Method}

\section{Design}

The study was a retrospective cross-sectional review of medical records for mother/infant dyads. Mother/ infant dyads were excluded for maternal conditions that 
may impact infant birth weight or weight loss, including maternal fever, diabetes (gestational and type I or II), placental abruption, chronic or pregnancy-induced hypertension, diuretics, fever, oligohydramnios, polyhydramnios, placenta previa, positive screen for drugs of abuse, renal or cardiac disease, rupture of membranes $\geq 24$ hours, sexually transmitted infections, and incomplete or inconsistent information.

Mother/infant dyads were excluded for infant conditions that may impact infant birth weight or weight loss, including birth weight $<2500$ or $>4000$ grams, gestational age $<37$ or $>42$ weeks, admission to Special Care Nursery, cardiac disease, congenital anomalies, gastrointestinal disease, thick meconium-stained fluid, phototherapy, renal disease, respiratory disease, sepsis, multiple birth, and incomplete or inconsistent information.

\section{Sample}

The study population was mothers and their infants who delivered at a Midwestern American hospital. The hospital has been certified continuously as a Baby Friendly Hospital ${ }^{\mathrm{TM}}$ since 2000. Initially the sample size of 200 mother/infant dyads was chosen to capture $10 \%$ of the 2000+ annual births. Based on the review of literature, it was expected to have $5 \%$ to $10 \%$ relative change in mean weight loss, with a standard deviation of $2 \%$ to $5 \%$. Considering the most conservative estimate of standard deviation as 5 with absolute deviation in mean weight loss as $15 \%$ of mean, with $5 \%$ level of significance, the estimated minimum sample size was determined to be 174 . A total of 518 mother/infant dyads were reviewed during December 2008 and April 2009 until 200 met the study objectives.

\section{Procedures}

A data-collection tool was utilized by the study investigators to review electronic and paper medical records, and record maternal demographics, prenatal history, labor and delivery, and IV intake; and infant demographics and weights throughout hospital admission of two-to-four days.

To minimize missing data and increase validity, maternity nursing staff were informed of the study and encouraged to make extra efforts to accurately chart IV volume and start/end times. Unclothed infants were weighed at birth, and on each additional in-patient hospital day during the 12-hour night shift. Digital infant scales were calibrated by the hospital clinical engineering department every two weeks during the study, and all scales remained accurate.
Ethical Considerations and Protection of Human Subjects

The study was approved by the University of Cincinnati Institutional Review Board and the Mercy Hospital Fairfield Medical Staff Quality Committee.

\section{Statistical Analysis}

The primary outcome measure was maximum percent weight loss from the birth weight on any subsequent daily weight during hospital stay (2, 3, or 4 days). The quantitative variables are described using mean and standard deviation (SD). Categorical variables are described using frequency and proportions. Non-normal variable, average IV fluid $\mathrm{mL} /$ hour, was transformed into log scale to induce normality needed to satisfy for parametric data analysis. Unadjusted ordinary linear regression was used to select important cofactors for multiple ordinary linear regression analysis. All the significant cofactors at $5 \%$ level of significance in the univariate analysis were included in multivariable analysis.

Stepwise multiple linear regression was used to assess factors associated with maximum weight loss with 5\% level of entry, and 10\% level of stay in the model. Firstorder interaction effect was explored for some of the important cofactors before finalizing the model. However, no interaction effect was found to be significant. Variables with variance inflation factor more than 10 were considered as collinear.

Model performances were described using $R^{2}$ statistics. Univariate results are reported using unadjusted regression coefficient (URC), with their 95\% confidence intervals $(\mathrm{CI})$ and adjusted results are reported using adjusted regression coefficient (ARC) with their 95\% CI. All the results with less than 5\% were considered as significant results. A scatter plot was constructed to show the relationship between IV fluids per hour and maximum infant weight loss (see below).

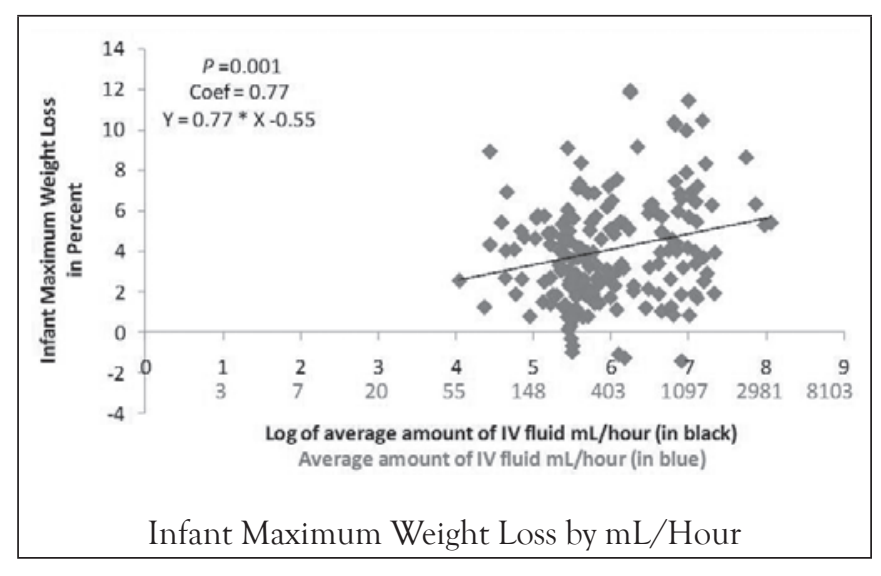




\section{Results}

A total of 200 subjects met the inclusion criteria and were included in the study. Out of 200, 14 mothers did not receive IV fluid. Coefficient of variation of subjects who received IV fluid was $57 \%$, and those who did not as $63 \%$. During analysis, the 14 mothers who did not receive any IV fluid were excluded resulting in $\mathrm{n}=186$. Mean maximum weight loss was 4.08\% (SD: 2.57).

Maternal demographics, prenatal history, labor-anddelivery statistics are described in Table 1 [link to Table 1]. In the univariate analysis, mother's age, marital status, ethnicity, insurance status, induction, augmentation, medication use during labor and delivery, anesthesia, method of delivery, feeding method, and IV fluid per hour were found to be significantly associated with maximum weight loss. For every one percent increase in average $\mathrm{mL}$ per hour, the infant maximum weight loss percent will increase by 0.0077 (see below).

\section{Example for Infant Maximum Weight Loss Percent}

For every one percent increase in average $\mathrm{mL}$ per hour, the infant maximum weight-loss percent will increase by 0.0077 . For example, assume a $5 \%$ infant maximum weight loss, and a change in average IV $\mathrm{ml}$ per hour from 150 to 225 (a 50\% increase). Then maximum weight-loss percent would be predicted to increase by $0.0077 \times 50$, which is 0.39 , giving a total maximum weight-loss percent of $5.39(5+0.39)$.

The variance inflation factor was found to be more than 10 for three variables (medication used during labor and delivery, anesthesia, and method of delivery). This indicates these three variables are highly collinear. Thus, we developed various multivariable regression models after including and excluding collinear variables. After including all collinear variables, in multivariable analysis, race, medications during labor and delivery, and feeding methods remained significant. These variables explained $46 \%\left(R^{2}=0.46\right)$ of the variability in the maximum percent weight loss.

Infants breastfeeding with supplements had significantly less weight loss when compared to those exclusively breastfed after adjusting other significant cofactors. After excluding medications during labor and delivery, anesthesia, and method of delivery, IV fluid was found to be one of the most significant factors associated with maximum weight loss after adjusting for feeding method (Table 1). This model explains $28 \%$ of the variability in the maximum weight loss $\left(R^{2}=0.28\right)$.

\section{Discussion}

Maternal average IV $\mathrm{mL}$ per hour positively correlated with infant maximum weight loss. However, there are many factors related to infant weight loss, and if an infant's weight loss is excessive, then additional assessments are indicated including, but not limited to:

\section{Maternal Assessment}

- Maternal IV fluid during labor

- Stage of lactogenesis

- Risk factors for breastfeeding complications

\section{Infant Assessment}

- Confirm current weight loss

- Repeat weight check in 12 to 24 hours

- Evaluate infant feeding frequency and effectiveness

- Evaluate infant hydration status

- Evaluate infant output

Although limited research exists in this area, preliminary results among current studies are trending to include maternal IV fluids in the assessment of an infant's weight loss in the first days of life before ordering supplementation.

\section{Recommendations}

Limitations of this study are primarily methodology related. Additional research is needed to confirm correlation between maternal IV fluid and infant weight loss. Enhancements to this study design may include: a) multi-site study to increase external validity, especially including hospitals without Baby Friendly certification, and with different racial and socioeconomic population; b) prospective method to increase data-collection accuracy; c) reduced exclusion criteria to increase ability to apply to larger populations; d) standardized infant weight checks to $24,48,72$ hours ( \pm 2$)$; and e) increased sample size.

\section{Acknowledgements}

This study was supported by an Institutional Clinical and Translational Science Award, NIH/NCRR Grant Number 5UL1RR026314-03. Its contents are solely the responsibility of the authors and do not necessarily represent the official views of the NIH.

The authors would like to thank Linda Baas, Athena Farmer, John (Chuck) Schafer, Rakesh Shukla, and Michele Stokes. 


\section{References}

Academy of Breastfeeding Medicine. (2009). ABM clinical protocol \#3: Hospital guidelines for the use of supplementary feedings in the healthy term breastfed neonate, revised 2009. Breastfeeding Medicine, 4(3), 175-182. doi:10.1089/bfm.2009.9991

American Academy of Pediatrics. (2012). Policy statement. Breastfeeding and the use of human milk. Pediatrics, 129(3), e827-e841. doi: $10.1542 /$ peds.2011-3552

Chantry, C., Nommsen-Rivers, L., Peerson, J., Cohen, R., \& Dewey, K. (2011). Excess weight loss in first-born breastfed newborns relates to maternal intrapartum fluid balance. Pediatrics, 127(1), e171-e179.

Dewey, K., Nommsen-Rivers, L., Heinig, M., \& Cohen, R. (2003). Risk factors for suboptimal infant breastfeeding behavior, delayed onset of lactation, and excess neonatal weight loss. Pediatrics, 112(3), 607-619.

International Lactation Consultants Association. (2005). Clinical guidelines for the establishment of exclusive breastfeeding, 2 nd edition. Raleigh, NC: Author.

Lamp, J., \& Macke, J. (2010). Relationships among intrapartum maternal fluid intake, birth type, neonatal output, and neonatal weight loss during the first 48 hours after birth. JOGNN: Journal of Obstetric, Gynecologic, EE Neonatal Nursing, 39(2), 169-177.

Martens, P., \& Romphf, L. (2007). Factors associated with newborn in-hospital weight loss: Comparisons by feeding method, demographics, and birthing procedures. Journal of Human Lactation, 23(3), 233-241.
Merry, H., \& Montgomery, A. (2000). Do breastfed babies whose mothers have had labor epidurals lose more weight in the first 24 hours of life? ABM NEWS and VIEWS, 6(3), 21. Retrieved from http://www.draparrilla.com/pdf/abstracts\%20WIC.pdf

Mulder, P., Johnson, T., \& Baker, L. (2010). Excessive weight loss in breastfed infants during the postpartum hospitalization. JOGNN: Journal of Obstetric, Gynecologic, $\mathcal{E}$ Neonatal Nursing, 39(1), 15-26.

Noel-Weiss, J., Woodend, K., Peterson, W., Gibb, W., \& Groll, D. (2011). An observational study of associations among maternal fluids during parturition, neonatal output, and breastfed newborn weight loss. International Breastfeeding Journal, 6:9. doi: 10.1186/1746-4358-6-9

Saki, A., Eshraghian, M., Mohammad, K., Foroushani, A., \& Bordbar, M. (2010). A prospective study of the effect of delivery type on neonatal weight gain pattern in exclusively breastfed neonates. Neonatal Intensive Care, 23(3), 52-54.

Sheehan, K. (2009). The role of intrapartum intravenous therapy and method of delivery on newborn weight loss: Challenging the 7\% rule. Master thesis. Retrieved from http://dr.library.brocku.ca/ bitstream/handle/10464/2925/Brock_Sheehan_Kim_2009. pdf?sequence $=1$

Vazirinejad, R., Darakhshan, S., Esmaeili, A., \& Hadadian, S. (2009). The effect of maternal breast variations on neonatal weight gain in the first seven days of life. International Breastfeeding Journal, 4:13. Retrieved from: http://www. internationalbreastfeedingjournal.com/content/4/1/13

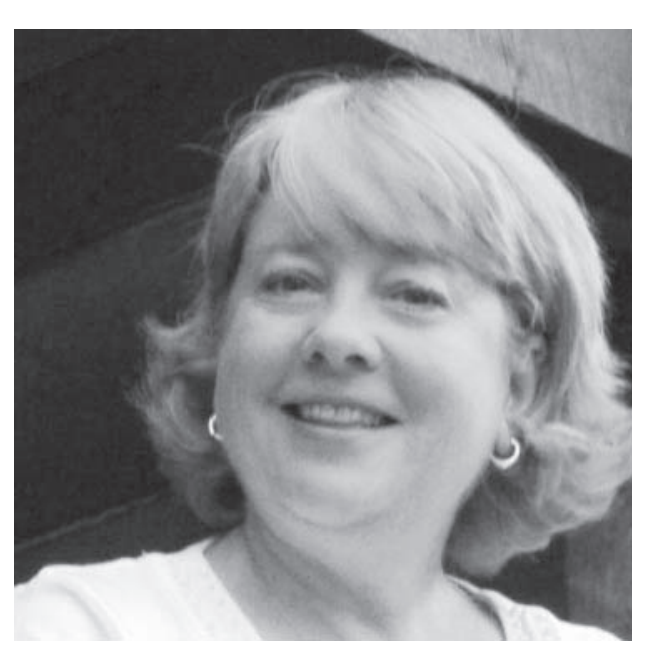

Robin Hirth, M.Ed., B.S., IBCLC, RLC is a board certified lactation consultant with 30 years experience working with breastfeeding families. She currently works as lactation consultant at Mercy Health-Fairfield Hospital in the Cincinnati, Ohio area. She planned and implemented the strategic plan, which qualified Mercy Hospital Mt. Airy in Cincinnati for the Baby Friendly Hospital ${ }^{\mathrm{TM}}$ Award in 1997. Her many volunteer efforts include speaking at local, regional, and national conferences; and serving as La Leche League Leader, IBLCE Exam Proctor, and Editorial Review Board Member for Clinical Lactation and the Journal of Human Lactation. 

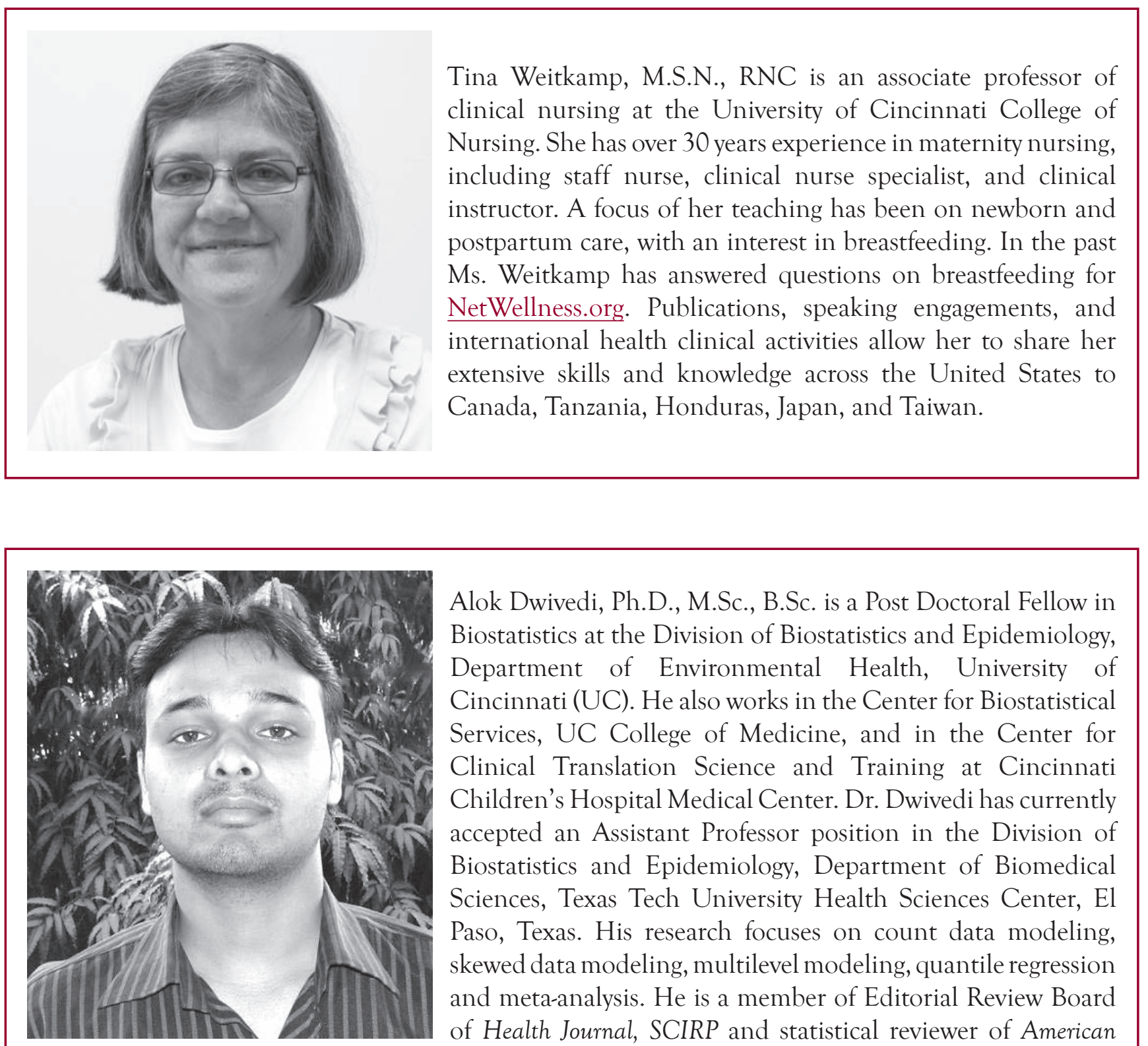

Alok Dwivedi, Ph.D., M.Sc., B.Sc. is a Post Doctoral Fellow in Biostatistics at the Division of Biostatistics and Epidemiology, Department of Environmental Health, University of Cincinnati (UC). He also works in the Center for Biostatistical Services, UC College of Medicine, and in the Center for Clinical Translation Science and Training at Cincinnati Children's Hospital Medical Center. Dr. Dwivedi has currently accepted an Assistant Professor position in the Division of Biostatistics and Epidemiology, Department of Biomedical Sciences, Texas Tech University Health Sciences Center, El Paso, Texas. His research focuses on count data modeling, skewed data modeling, multilevel modeling, quantile regression and meta-analysis. He is a member of Editorial Review Board of Health Journal, SCIRP and statistical reviewer of American Heart Journal and Circulation. Dr. Dwivedi also participates in reviewing intramural and methodological proposals. 